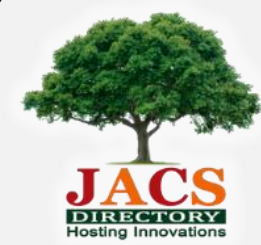

Journal of Nanoscience and Technology

\title{
Nano-Biosensors for Cancer Diagnostics: Current Status and Future Prospects
}

\author{
Kanchan Karki ${ }^{1}$, , Rashmi Bisht ${ }^{1}$, K.S. Shahi², Rinku Ranga ${ }^{3}$, Uzra Malik ${ }^{3}$ \\ ${ }^{1}$ Molecular Diagnostic and Nanotechnology Laboratory, Uttarakhand Council for Biotechnology, U.S. Nagar - 263 146, Uttarakhand, India. \\ ${ }^{2}$ Department of Surgery, Dr. Shushila Tiwari Medical College, Haldwani, Nainital - 263 139, Uttarakhand, India. \\ ${ }^{3}$ Devsthali Vidyapeeth, Kumaun University, Nainital - 263 139, Uttarakhand, India.
}

\section{A R T I C LEDE T A I L S}

\section{Article history:}

Received 21 May 2019

Accepted 06 June 2019

Available online 13 June 2019

\section{Keywords:}

Cancer Biomarkers

Nano-Biosensors

Nanomaterials

\begin{abstract}
A B S T R A C T
Cancer is one of the leading life-threatening diseases all over the globe which costs highest mortality rates. Early detection of cancer increases the chance of a cure and survival rates but unfortunately mostly cancers are diagnosed only after they have metastasized throughout the body. The list of the biomarkers associated with over 200 types of cancer has witnessed a significant increase in the recent past however the highly sensitive and specific biomarkers are still need of time. Biosensors are devices that are designed to detect a specific biological analyte by converting the complex biological reactions into an electrical signal whose intensity is associated with the concentration of the analyte. Nanotechnology with nanomaterials improves and modifies the biorecognition element part to improve the biosensing phenomenon and makes it one of the hottest topics attracting scientific fraternity. Use of diverse numbers of nanomaterials with good electrical and sensing properties has promised a smarter and faster detection with better reproducibility. This review briefly summarizes the few different types of biosensors based on different types of nanomaterials for cancer biomarker detection. This paper also highlights the obstacles related to their developmental and implicational aspects.
\end{abstract}

\section{Introduction}

Cancer by definition is an abnormal and uncontrolled cell growth due to an accumulation of specific genetic and epigenetic defects. It may have both environmental and hereditary origin. Unregulated cell growth leads to the formation of a tumor mass that over time loses its control over normal homeostatic checks and balances [1]. As the cancer progresses and metastasizes to other body organs and systems it becomes more and more untreatable. In general cancer is a multifactorial disease, and includes a complex range of genetic and epigenetic alterations which disturb the cellular microenvironment which is associated with the onset and progression of disease $[2,3]$.

According to the recent report of the national cancer institute on cancer statistics an estimated 1,735,350 new cases of cancer were diagnosed in the United States and 609,640 people died from the disease in 2018 [4]. The most common cancers in descending order are breast cancer, lung and bronchus cancer, prostate cancer, colon and rectum cancer, melanoma of the skin, bladder cancer, non-Hodgkin lymphoma, kidney and renal pelvis cancer, endometrial cancer, leukemia, pancreatic cancer, thyroid cancer, and liver cancer. Approximately $38.4 \%$ of men and women will be diagnosed with cancer at some point during their lifetimes (based on 2013-2015 data) [4]. Despite of recent technological advancements and new treatment modalities late diagnosis and poor prognosis are still the primary reasons for poor survival rate of cancer patients. This review paper summarizes recent progress in the development of nano-biosensors by integrating functional bio-molecules with different types of nanomaterials, including carbon nanotubes, graphene, carbon dots etc. We will also envision nanosensors to make significant improvements in the cancer diagnosis with onsite point-of-care devices.

\section{Role of Biomarkers in Cancer Diagnosis}

The most popular advancement in the field of cancer diagnostics that have come to the fore was the development of a test for first cancer biomarker i.e cacinoembrionic antigen (CEA) detection by Dr. Joseph Gold in 1965 [5]. A number of potential serum test have been developed for a variety of cancers by now. Cancer biomarkers are key indicators of tumor growth. They are used not only to diagnose and monitor disease, but also to provide a prognostic approach to treatment [6]. The biomarkers by definition are the molecules which undergo important alterations in their levels during cancer onset progression and treatment. Biomarkers may be proteins, enzymes, nucleic acids, antibodies and antigens which are classified in various categories such as diagnostic, prognostic, biomarkers to set treatment modality etc., [7]. Diagnostic biomarkers are used for the detection of the disease, whereas the information about course of recurrence of the disease is given by prognostic biomarkers $[8,9]$. Precise detection and analysis of these biomarkers could help in early detection of disease, disease progression and to check the response of treatment modalities [10]. However, there are only few specific biomarkers which can be used in clinical setting e.g. Prostate Specific Antigen (PSA) for prostate cancer screening and monitoring, cancer antigen 15.3 (CA 15.3), estrogen receptor (ER), progesterone receptor (PR), cancer antigen 27.29 (CA27.29) for breast cancer monitoring, bladder tumor antigen (BTA) for bladder cancer monitoring etc. whereas others can be related to more than one type of diseases.

\subsection{Disadvantages of Conventional Methods of Cancer Detection}

The most common imaging method used to monitor is Computed Tomography (CT), which provides cross-sectional imaging by computer using X-rays. Magnetic Resonance Imaging (MRI) is a procedure that uses potent magnetic fields to detect the abnormalities $[11,12]$. Ultra sound examination is always useful for examining the cervix, pancreas, liver and kidneys [13]. Endoscopic examinations are usually used to investigate the gastrointestinal tract, bronchial tubes, cervix, prostrate, bladder or head and neck section [14]. Mammography is mainly used to treat breast cancer screenings. In the isotopic diagnostics, a radioactive tracer is established into the patient's body [15]. The marker goes to the organ to be examined used to classify the occurrence of various cancers such as breast, prostate and colorectal cancer $[16,17]$. The most familiar problem associated with these methods of diagnosis is the high risk of false positives. Magnetic resonance imaging (MRI), one of the most common imaging technologies used today for cancer diagnosis and monitoring, cannot detect entities that are smaller than a few centimeters. The use of nanomaterials as imaging agents allows for more sensitive and precise measurement of cancerous tissues $[18,19]$. 
Tissue biopsy is commonly used in the clinic to diagnose a variety of cancers. It plays an important role in tailoring best treatment options for the individuals at diagnosis. However, tissue-based biopsy is invasive, expensive, impractical for repeated testing, and unavailable to some cancer types. For example, over $30 \%$ of advanced non-small cell lung cancers do not have accessible tissue [20]. Especially, tumors are heterogeneous and evolve over time. Thus, biopsy data from the limited amount of collected tissues are often biased and can provide false results.

The limitations in tissue biopsy have driven the development of liquid biopsy, a means to sense cancer biomarkers in body fluids such as blood, serum, saliva and urine to aid in cancer screening, diagnosis and treatment [21]. Liquid biopsy is comparatively non-invasive, inexpensive, accessible to large populations, and allows for repeated testing for realtime monitoring of disease stage and treatment effectiveness. One of the major advantages related to bio fluids is that it contains information from all the tumour cells in the patient rather than small portion of a tumour in the tissue biopsy [22]. The regular methods used for biofluids detection are the traditional enzyme-linked immunosorbent assay (ELISA) or Polymerase chain reaction (PCR) based methods. The technological limitations of these methods are consumption of expensive reagents in every assay, slow detection, instrument complexity and requirement of trained personnel [23].

Also, being manual techniques, these methods are not capable in the continuous monitoring of the patient during treatment. Besides, within the cell multiple events are associated with all cancers involving more than one molecule. Thus, simultaneous detection of multiple biomarkers for correct diagnosis and prognosis is required [24]. Besides that, some additional traditional methods such as northern blot, southern blot analysis and other blotting techniques suffer low sensitivity, time consumption and high cost. Therefore, there is an urgent need for rapid, reliable, specific and sensitive modified alternative technique for cancer detection.

\section{Definition and Conceptual Idea of Biosensors}

Accurate and early diagnosis of cancer has the potential to reduce the rate of mortality and morbidity of cancer patients. Cancer biomarkers can be used for screening, monitoring treatment progression and fix treatment modalities. Biosensors are the devices which operate on the theory of signal transduction [25]. The components of the biosensor include a biorecognition element, a biotransducer and an electronic system composed of a display, processor and amplifier. The bio-recognition element may be a bioreceptor, antibody or enzyme which is allowed to interact with their corresponding particular analyte [26,27]. The transducer measures this interaction and amplifier amplified the signal to the limit of detection and outputs a signal. The strength of the signal output is directly proportional to the concentration of the analyte. The signal is then improved and processed by the electronic system and the unknown analyte is recognized successfully [28]. In recent years researchers have shown a keen interest in fabrication of cancer biosensors due to their greater analytical performance and real-time measurement of particular analyte [29,30]. A simple schematic diagram of the components of the general biosensor viz. bioreceptor, transducer, and detector are depicted in Fig. 1.

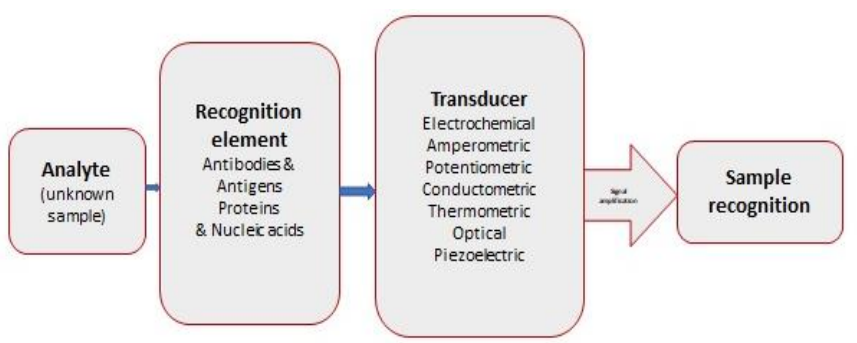

Fig. 1 This schematic diagram represents how an unknown analyte captured by the recognition element part induces a change in the transducer's surface. The transducer transforms these recognition events into a detectable signal, which can be used for the quantification or determination of the analyte

\subsection{Classifications of Biosensors}

There are different types of biosensors based on the sensor devices and the biological materials and some of them are demonstrated in Table 1. In recent years, these biosensors have become very popular, and they are applicable in different fields such as healthcare, agricultural, and veterinary applications, drug improvement, industrial and defense processing and monitoring etc. however these devices are still not so common in cancer detection. Fig. 2 represents the areas of biosensor applications.

https://doi.org/10.30799/jnst.260.19050415
Table 1 Classification of general biosensors based on their working principals

\begin{tabular}{|c|c|c|}
\hline S. No. & Type of Biosensor & Principle \\
\hline 1. & Electrochemical Biosensor & $\begin{array}{l}\text { Utilizes the principle of the measurement } \\
\text { of electronic current by electrodes }\end{array}$ \\
\hline 2. & Amperometric Biosensor & $\begin{array}{l}\text { Utilizes the principle of electronic current } \\
\text { determination as a reaction of enzyme- } \\
\text { catalyzed redox reaction }\end{array}$ \\
\hline 3. & Potentiometric Biosensor & $\begin{array}{l}\text { Utilizes the principle of changes the } \\
\text { concentration of ions which is determined } \\
\text { by the ion-selective pH electrodes }\end{array}$ \\
\hline 4. & Conductometric Biosensor & $\begin{array}{l}\text { Utilizes the principle of measurement of } \\
\text { drastic alterations in ionic concentration }\end{array}$ \\
\hline 5. & Thermometric Biosensor & $\begin{array}{l}\text { Utilizes the principle of biological reactions } \\
\text { which are connected with the production } \\
\text { of heat }\end{array}$ \\
\hline 6. & Optical Biosensors & $\begin{array}{l}\text { Utilizes the principle of optical } \\
\text { measurements like fluorescence, } \\
\text { absorbance and etc. }\end{array}$ \\
\hline 7. & Piezoelectric Biosensors & $\begin{array}{l}\text { Utilizes the principle of measurement of } \\
\text { alteration in the response frequencies } \\
\text { using piezoelectric crystals }\end{array}$ \\
\hline 8. & Immuno-Biosensors & $\begin{array}{l}\text { Utilizes the principle of immunological } \\
\text { specificity and mostly coupled with } \\
\text { measurement on the Potentiometric } \\
\text { Biosensors. }\end{array}$ \\
\hline
\end{tabular}

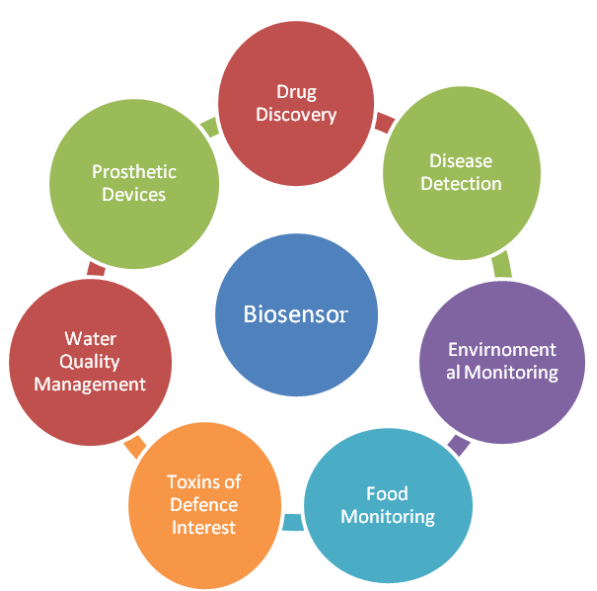

Fig. 2 Different areas of applications of biosensors

\section{Definition and Conceptual Idea of Nanobiosensor}

When we merge the understanding of biosensor concepts with the highly promising field of nanotechnology it develops a new area of disease diagnostics i.e. nanodiagnostics [31]. In this area we pay an attention to the design and modification of nanomaterials for improving cancer detection and treatment. Huge numbers of nanoparticles are currently under development for applications related to cancer diagnosis [32].

The miniature devices developed by the rapidly emerging field of nanotechnology improves the chances of detecting cancer earlier, thus improving patient survival rates. Several types of nanomaterials have added abundant advantages over traditional diagnosis [33, 34]. Nanomaterial have few unique properties such as high electrical conductivity, high surface to volume ratio, better shock bearing ability and the enhanced signal responses which makes them most suitable for biosensor technology. The classifications based on dimensions are mentioned in Table 2 and the unique properties of few nanomaterials has been discussed in a separate section below.

Table 2 Classification of nanomaterials based on dimensions

\begin{tabular}{lll}
\hline S. No. & $\begin{array}{l}\text { Dimensions of } \\
\text { nanomaterials }\end{array}$ & Examples \\
\hline 1. & One dimensional (1D) & Nanotubes, Nanorods, Nanowires \\
2. & Two dimensional (2D) & Graphene, Nanofilms, Nanolayers, Nanocoatings \\
3. & Three dimensional & Bulk Powders, Bundles of Nanowires, Nanotubes, \\
& (3D) & Multi-nanolayers \\
\hline
\end{tabular}

\subsection{Classifications of Nanobiosensors}

The classification of nanobiosensors is a very diverse area and it is not as simple as it is in the case of general biosensors. The classification of the nanobiosensor is based on the nature of nanomaterials incorporated in the 
biosensing operation. Table 3 briefs the advantages of currently used nanomaterials and in this section, we will discuss the properties of few different nanomaterials which have been extensively studied for fabrication of nanobiosensors and cancer diagnosis so far.

Table 3 List of few extensively used nanomaterials with their brief applications

\begin{tabular}{|c|c|c|}
\hline S.No. & Nanoplateform & Application \\
\hline \multirow[t]{4}{*}{1.} & Carbon & Improved enzyme loading \\
\hline & Nanotubes & Have higher aspect ratios \\
\hline & & Have ability to be functionalized \\
\hline & & Better electrical communication \\
\hline \multirow[t]{3}{*}{2.} & Quantum Dots & Have excellent fluorescence \\
\hline & & Have quantum confinement of charge carriers \\
\hline & & Have Size tunable band energy \\
\hline \multirow[t]{3}{*}{3.} & Nanowires & Highly versatile \\
\hline & & $\begin{array}{l}\text { Have good electrical and sensing properties for bio- and } \\
\text { chemical sensing }\end{array}$ \\
\hline & & Better charge conduction \\
\hline \multirow[t]{3}{*}{4.} & Nanoparticles & Better immobilization efficiency \\
\hline & & Better loading efficiency \\
\hline & & Better catalytic properties \\
\hline \multirow[t]{3}{*}{5.} & Nanorods & Good plasmonic materials \\
\hline & & Size tunable energy regulation \\
\hline & & It can be coupled with MEMS \\
\hline
\end{tabular}

\subsection{Quantum Dots Based Sensors for Cancer Detection}

Quantum dots (QDs) are semiconductor nanocrystals which generally range from $2 \mathrm{~nm}$ to $10 \mathrm{~nm}$ in diameter. With this special size and the optical and chemical advantages of (QDs), QDs based theragnostic technology (Diagnostics + therapy) is rapidly emerging. QDs have improved imaging capabilities, brightness, and photostability and due to these characteristics, they may be used for simultaneous detection of multiple cancer biomarkers [35]. With outstanding biomedical imaging capabilities, QD-based imaging is emerging as one of the most promising biomedical imaging platforms for tumor microenvironment studies both in vivo and in vitro [36,37].

Since the first introduction of biocompatible QDs imaging of cancer cells in vitro in 1998for the first time [38,39], researchers have developed QD-based probes conjugated with cancer specific ligands, antibodies or peptides for cancer imaging and diagnosis in vitro [40,41]. Researchers demonstrated that QD-IHC is more accurate and precise at low protein expression levels compared with traditional immuno-histochemistry (IHC) technique and due to its high specificity and sensitivity it can achieve ultrasensitive detection which will provide much more information for personalized treatment options $[42,43]$. Although QDs have greater potential for cancer diagnosis, there are some toxicological and pharmacological disadvantages related to heavy metals toxicity and colloidal instability in vivo, which limits its advancement toward treatment of cancer and other diseases [44,45]. Keeping these observations in view there is an urgent need to develop QD's based diagnostic applications in vitro with minimally invasive techniques. In recent years scientists are putting efforts to generate novel QDs based nanobiosensors to maximize detection efficiency in vitro [46-48].

\subsection{Graphene Based Sensors for Cancer Detection}

The advantages conferred by the physical, optical and electrochemical properties of graphene- nanomaterials have vastly contributed to the current variety of ultrasensitive and ultraselective biosensor devices [49].

Graphene is a two-dimensional (2D) nanomaterial, which plays a tremendous role in the electronic and sensor communities due to its improved physical, optical and electrochemical properties [50,51]. Graphene is defined as "a single atom- thick sheet of hexagonally arranged, $\mathrm{sp}^{2}$ - bonded carbon atoms occurring within a carbon material structure. The nanothickness graphene film with $100 \mu \mathrm{m}$ of lateral size is observed as carbon planes connected together by van der Waals forces acting over a distance of about $0.335 \mathrm{~nm}[52,53]$. The properties of graphene are those of a semi - metal and are stable under ambient circumstances. Graphene can be engineered and chemically modified with migration of heteroatoms, oxidation, and reduction using inexpensive methods [54,55].

Chemical modifications such as oxidation and reduction can be incorporated in the single atom thick sheet graphene which can be further modified and functionalized [56,57]. The functionalized graphene allows immobilization of different nanoparticles (NPS), quantum dots (QDs), DNA, enzymes, protein, antigen, antibodies, and other specific molecules [58]. In medical diagnostic applications, graphene based biosensors have been recently fabricated with high sensitivity and specificity. Researchers developed a GFET biosensor for real time monitoring of carcinoembryonic https://doi.org/10.30799/jnst.260.19050415 antigen (CEA) with high sensitivity $(<100 \mathrm{pg} / \mathrm{mL}$ ) [59]. In another report human papillomavirus (HPV) was detected with the use of a novel paperbased graphene biosensor [60].

Since graphene material has been very well established in recent years, scientists are trying to explore other 2D materials with improved physical and chemical properties. These materials can be modified and incorporated in different biosensor platforms to give ultra-high sensitivity and selectivity which may contribute in providing a better solution to existing early diagnostic challenges [61].

\subsection{Carbon Nanotube Based Sensors for Cancer Detection}

CNTs are carbon allotropes. The structure of a carbon nanotube can be seen as a single and rolled up graphene sheet. CNTs can be divided into two main groups: Single-Walled Carbon Nano Tubes (SWCNTs), in which only a single graphene sheet is wrapped, or Multi-Walled Carbon Nano Tubes (MWCNTs), where more than one sheet is wrapped in a concentric fashion [62-64]. Diameters can range from a few nanometers up to hundreds of nanometers. Carbon nanotubes (CNTs) have been exploited for the development of electrochemical and biological sensors because of their excellent electrochemical properties, large surface area, ballistic electron transport and high mechanical strength [65]. Since the discovery of carbon nano tubes in 1990's, their extensive use in different techniques is growing due to their extraordinary attributes [66,67]. These excellent properties help researchers to exploit both single walled nanotubes as well as multi-walled nanotubes to fabricate biosensors for better diagnosis. The advantages conferred by the optoelectronic properties of carbon nanotubes have contributed to the recent variety of biosensor devices which makes them one of the most popular nanomaterials in the world of material science [68].

Recently most CNTs are used in drug delivery applications; however engineered CNTs can be used in different imaging techniques. These imaging capabilities together with right functionalization strategy can be used to detect reactive oxygen species (ROS) or specific antigens and specifically cancer markers [69]. Likewise, Graphene, right functionalization strategy is the most important and tedious step for CNTs [70]. Basically, two kinds of functionalization can be incorporated on CNTs: covalent for strong binding, but changed electrical properties or non-covalent for weak binding but preserved electrical properties [71]. The peculiar size carbon nanotubes along with unique physical and optical properties guide researchers for extensive exploration of CNTs in various cancer therapy and diagnostics applications.

\subsection{Future Prospects of Implementation of Nanobiosensors for Cancer Diagnosis}

Nanosensors have turn out to be an emerging area of interdisciplinary research in the recent years. Nanomaterials have enabled the development of ultrasensitive biosensors, because of their high surface area, electronic properties, and electrocatalytic activity as well as good biocompatibility. Within the next decade, nanodiagnostics will become available, and be able to perform thousands of biomarkers simultaneously, rapidly and inexpensively. The most common clinical diagnostic application will be analysis of biofluids by nanobiosensors.

Nanobiosensors can offer ultrasensitivity in detection of cancer biomarkers, which may be applied in the future for early detection as well as treatment of cancer. Nanodevices for this purpose are now in the feasibility stage. Nanotheranostics is an emerging area of nanotechnology where nanotheranotics devices will be implanted as a prophylactic measure in individuals who do not have any noticeable manifestations of cancer and monitoring could be conducted by smartphones. Such monitoring could detect cancers at the preliminary stages and enable healthcare professionals for suitable therapeutic intervention. Early detection and treatment would increase the chances of a cure. Future trends in diagnostics will continue in miniaturization of biochip technology to the nanoscale range.

\subsection{Challenges and Limitations of Nanobiosensors}

Despite being launched decades ago, glucose meter and lateral flow pregnancy tests remain the most commercialized biosensors [72]. In recent years, several biosensors taking advantage of nanomaterials have been developed but unfortunately it generated enormous amount of published literature only. Practically it could not be moved forward in ground level with potential applicability. Developed biosensing strategies are technically complicated and unable to solve the practical diagnostic problems in real life. Therefore, there commercialization is not feasible. There is one more issue which limits the development and application of nanobiosensors in real life is safety and toxicity related to nanomaterials. It is still a part of controversial discussions which requires further studies related to these materials and their special effects. 


\section{Conclusion}

In conclusion we can articulate that nanotechnology has the enormous potential to revolutionize the case of cancer detection. As the transducer part of the nanobiosensors are significantly improved and modified with the use of nanomaterials and nanostructures for biological recognition element immobilization, it provides a better platform for smarter and quicker cancer detection. Besides their various advantages over the conventional cancer detection strategies such as their robustness and dynamic behavior, nanobiosensor development is still a very cumbersome process due to its technical complexities. To resolve these technical complexities there is an urgent need to advance the process of miniaturization of nanobiosensor devices so that point of care diagnosis can be done on site in a user-friendly way. Additionally, to target biosensors towards clinics, multitarget detection of multiple biomarkers simultaneously with high accuracy and reliability is very necessary. In case this target is achieved and these kinds of multitarget on-site testing miniature devices can be fabricated it will be very simple and easy to detect early cancers by minimally or completely non-invasive methods.

\section{References}

[1] B. Bohunicky, S.A Mousa, Biosensors: the new wave in cancer diagnosis, Nanotechnol. Sci. Appl. 4 (2010) 1-10.

[2] W.A. Flavahan, E. Gaskell, B.E. Bernstein, Epigenetic plasticity and the hallmarks of cancer, Science 357 (2017) (6348) 1-20.

[3] B.V. Chakravarthi, S. Nepal, S. Varambally, Genomic and Epigenomic alterations in cancer, Am. J. Pathol. 186 (2016) 1724-1735.

[4] N. Howlader, A.M. Noone, M. Krapcho, SEER cancer statistics review (CSR) (1975-2015), National Cancer Institute, Bethesda, MD, 2018.

[5] P. Gold, J. Shuster, O.S. Freedman, Carcinoembryonic antigen (CEA) in clinical medicine, Histor. Perspect. Pitfalls Projection 42 (1978) 1399-1405.

[6] S.K. Chatterjee, B.R. Zetter, Cancer biomarkers: knowing the present and predicting the future.Future Oncol. 1(2005) 37-50.

[7] V.S.P.K. Jayanthi, U. Saxena, A.B. Das, Recent advances in biosensor development for the detection of cancer biomarkers, Biosens. Bioelectron. 91 (2017) 15-23.

[8] Z.V. Fong, J.M. Winter, Biomarkers in pancreatic cancer: diagnostic, prognostic, and predictive, Cancer J. 18 (2012) 530-538.

[9] D.F. Hayes, R.C. Bast, C.E. Desch, Tumor marker utility grading system: a framework to evaluate clinical utility of tumor markers, J. Nat. Cancer. Inst. 88 (1996) 1456-1466.

[10] S.K. Chatterjee, B.R. Zetter, Cancer biomarkers: knowing the present and predicting the future, Future Oncol. 1 (2005) 37-50.

[11] A.J. Detre, Magnetic resonance imaging, Int. J. Basic Res. Clin. Appl. 73 (2019) 793-800.

[12] P. Lam, J. Marcin, What to know about MRI scans?, Med. News Today, 2018. (Retrieved from https://www.medicalnewstoday.com/articles/146309.php on 01.02.2019)

[13] A.E. Chu, J.Y. Kim, Laryngeal cancer: diagnosis and preoperative work-up, Otolaryn. Clin. North Am. 41 (2008) 673-695.

[14] J. Skowronek, Current status of brachytherapy in cancer treatment - short overview, J. Contemp. Brachyther. 6 (2017) 581-589.

[15] V.J. Frangioni, New technologies for human cancer imaging, J. Clin. Oncol. 26 (2008) 4012-4020.

[16] K.C. Kuhl, The changing world of breast cancer a radiologist's perspective, Invest. Radiol. 50 (2015) 615-628.

[17] B.S. Glass, A. Zeeshan, Clinical utility of positron emission mammography, Proc. Bayl. Univ. Med. Cent. 26 (2013) 314-319.

[18] R.K. Arora, R.P. Saini, Biosensors: way of diagnosis, Int. J. Pharm. Sci. Res. 4 (2013) 2517-2527.

[19] V. Jaishree, D.P Gupta, Nanotechnology: A revolution in cancer diagnosis, Indian J. Clin. Biochem. 27 (2012) 214-220.

[20] E.S. Kim, V. Hirsh, T. Mok, Gefitinib versus docetaxel in previously treated nonsmall-cell lung cancer (INTEREST): a randomised phase III trial, Lancet. 372 (2008) 1809-1818.

[21] G. Brock, E. Castellanos-Rizaldos, L. Hu, Liquid biopsy for cancer screening, patient stratification and monitoring, Transl. Cancer Res. 4 (2015) 280-290.

[22] Jr. LA Diaz, A. Bardelli, Liquid biopsies: genotyping circulating tumor, J. Clin. Oncol. 32 (2014) 579-586.

[23] S. Kumar, A. Mohan, R. Guleria, Biomarkers in cancer screening, research and detection: present and future: a review, Biomark. 11 (2006) 385-405.

[24] M. Bustoros, H.T. Mouhieddine, A. Detappe, Established and novel prognostic biomarkers in multiple myeloma, Am. Soc. Clin. Oncol. 37 (2017) 548-560.

[25] B. Bohunicky, S.A. Mousa, Biosensors: the new wave in cancer diagnosis, Nanotechnol. Sci. Appl. 4 (2011) 1-10.

[26] N. Bhalla, P. Jolly, N. Formisano, Introduction to biosensors, Essay. Biochem. 60 (2016) 1-8.

[27] S. Malhotra, A. Verma, N. Tyagi, Biosensors: Principle, types and applications, Int. J. Adv. Res. Innovat. Ideas Edu. 3 (2017) 3639-3644.

[28] N.S. Ramgir, Electronic nose based on nanomaterials, issues, challenges, and prospects, ISRN Nanomater. 2013 (2013) 1-22.

[29] $\mathrm{Wu} \mathrm{Li,} \mathrm{X.} \mathrm{Qu,} \mathrm{Cancer} \mathrm{biomarker} \mathrm{detection:} \mathrm{recent} \mathrm{achievements} \mathrm{and} \mathrm{challenges,}$ Chem. Soc. Rev. 4 (2015) 2963-2997.

[30] P. Mehrotra, Biosensors and their applications - A review, J. Oral Biol. Craniofac. Res. 6 (2016) 153-159.

https://doi.org/10.30799/jnst.260.19050415
[31] K.M. Koo, P.N. Mainwaring, S.A. Tomlins, Merging new-age biomarkers and nanodiagnostics for precision prostate cancer management, Nature Rev. Urology 16 (2019) 302-317.

[32] S.C. Baetke, T. Lammers, F. Kiessling, Applications of nanoparticles for diagnosis and therapy of cancer, Br. J. Radiol. 88 (2015) 20150207:1-12.

[33] H.M. Azzazy, M.M. Mansour, S.C. Kazmierczak, Nanodiagnostics: A new frontier for clinical laboratory medicine, Clin. Chem. 52 (2006) 1238-1246.

[34] Y.H. Yun, E. Eteshola, A. Bhattacharya, Tiny medicine: Nanomaterial-based biosensors, Sensors (Basel) 9 (2009) 9275-9299.

[35] Y. Hu, D.H. Fine, E. Tasciotti, Nanodevices in diagnostics, Wiley Interdiscip. Rev. Nanomed. Nanobiotechnol. 3 (2011) 11-32.

[36] A.P. Alivisatos, W. Gu, C. Larabell, Quantum dots as cellular probes, Ann. Rev. Biomed Eng. 7 (2005) 55-76.

[37] A.M. Smith, H. Duan, A.M. Mohs, Bioconjugated quantum dots for in vivo molecular and cellular imaging, Adv. Drug Deliv. Rev. 60 (2008) 1226-1240.

[38] H. Zhang, D. Yee, C. Wang, Quantum dots for cancer diagnosis and therapy: biological and clinical perspectives, Nanomed. (Lond.) 3 (2008) 83-91.

[39] W.C. Chan, S. Nie, Quantum dot bioconjugates for ultrasensitive nonisotopic detection, Science 281 (1998) 2016-2018.

[40] M.Jr. Bruchez, M. Moronne, P. Gin, Semiconductor nanocrystals as fluorescent biological labels, Science 281(1998) 2013-2016.

[41] X. Wu, H. Liu, J. Liu, Immunofluorescent labeling of cancer marker Her2 and other cellular targets with semiconductor quantum dots, Nat. Biotechnol. 21 (2003) 41-46.

[42] Z. Li, K. Wang, W. Tan, Immunofluorescent labeling of cancer cells with quantum dots synthesized in aqueous solution, Anal. Biochem. 354 (2006) 169-174.

[43] C. Chen, J. Peng, S.R. Sun, Tapping the potential of quantum dots for personalized oncology: current status and future perspectives, Nanomed. (Lond.) 7 (2012) 411-428.

[44] C. Chen, S.R. Sun, Y.P. Gong, Quantum dots-based molecular classification of breast cancer by quantitative spectroanalysis of hormone receptors and HER2, Biomater. 32 (2011) 7592-7599.

[45] R. Hardman, A toxicologic review of quantum dots: toxicity depends on physicochemical and environmental factors, Environ. Health Perspect. 114 (2006) 165-172.

[46] B.A. Rzigalinski, J.S. Strobl, Cadmium-containing nanoparticles: perspectives on pharmacology and toxicology of quantum dots, Toxicol. Appl. Pharmacol. 238 (2009) 280-288.

[47] X. Michalet, FF. Pinaud, L.A. Bentolila, Quantum dots for live cells, in vivo imaging, and diagnostics, Science 307 (2005) 538-544.

[48] H.S. Choi, W. Liu, F. Liu, Design considerations for tumour-targeted nanoparticles, Nat. Nanotechnol. 5 (2010) 42-47.

[49] M. Bottrill, M. Green, Some aspects of quantum dot toxicity, Chem. Commun (Camb.) 47 (2011) 7039-7050.

[50] P. Suvarnaphaet, S. Pechprasarn, Graphene-based materials for biosensors: A Review, Sensors (Basel.) 17 (2017) 1-24.

[51] M. Zhang, A. Halder, X. Cao, Graphene paper based electrochemical sensors, Electrochem. Sensors Technol. Tech Open 2017 (2017) 33-62.

[52] F. Nada, Atta, Ahmed, Galal, H. Ekram, El- Ads, Graphene- A platform for sensor and biosensor applications, Biosens. Micro Nanoscale Appl. 4 (2015) 37-84.

[53] J. Choi, C.M. Wang, S.Y.R. Cha, Graphene bioelectronics, Biomed. Eng. Lett. 3 (2013) 201-208.

[54] M. Skoda, I. Dudek, A. Jarosz, Graphene: one material, many possibilitiesapplication difficulties in biological systems, J. Nanomater. 2014 (2014) 1-12.

[55] W. Tian, W. Li, W. Yu, A review on lattice defects in graphene: Types, generation, effects and regulation, Micromachines 8 (2017) 163:1-15.

[56] M.J. McAllister, J.L. Li, D.H. Adamson, Single sheet functionalized graphene by oxidation and thermal expansion of graphite, Chem. Mater. 19 (2007) 43964404.

[57] J. Liu, J. Tang, J.J. Gooding, Strategies for chemical modification of graphene and applications of chemically modified graphene, J. Mater. Chem. 22 (2012) $12435-12452$.

[58] A. Samanta, I.L. Medintz, Nanoparticles and DNA - a powerful and growing functional combination in bionanotechnology, Nanoscale 8 (2016) 9037-9095.

[59] V.B. Chikkaveeraiah, A. Bhirde, Y.N. Morgan, Electrochemical immunosensors for detection of cancer protein biomarkers, ACS Nano 6 (2012) 6546-6561.

[60] P. Teengam, W. Siangproh, A. Tuantranont, Electrochemical paper-based peptide nucleic acid biosensor for detecting human papillomavirus, Anal. Chim. Acta. 952 (2017) 32-40.

[61] L.Y.M. Sin, E.K. Mach, K.P. Wong, Advances and challenges in biosensor-based diagnosis of infectious diseases, Expert Rev. Mol. Diagn. 14 (2014) 225-244.

[62] N. Saifuddin, A.J. Raziah, A.R. Junizah, Carbon nanotubes: A review on structure and their interaction with proteins, Jour. Chem. 2013 (2013) 1-18.

[63] C.R. Jat, K. Arora, N. Anuragi, Carbon nanotubes: advancement in science and technology, World J. Pharm. Pharm. Sci. 4 (2015) 1533-1550.

[64] S. Nasir, M.Z. Hussein, Z. Zainal, Carbon-based nanomaterials/allotropes: A glimpse of their synthesis, properties and some applications. Mater. (Basel.) 11 (2018) 1-24.

[65] X. Zhang, Q. Guo, D. Cui, Recent advances in nanotechnology, Biosensors. Sensors (Basel.) 9 (2009) 1033-1053.

[66] A. Aqel, K.M. AbouEl-Nour, A.A. Ammar, Carbon nanotubes, science and technology part (I) structure, synthesis and characterization, Arab. J. Chem. 5 (2012) $1-23$.

[67] V. Sgobba, M.D. Guldi, Carbon nanotubes- electronic/electrochemical properties and application for nanoelectronics and photonics, Chem. Soc. Rev. 38 (2009) 165-184.

[68] A. Chinnappan, B. Chinnappan, H. Kim, Carbon nanotube hybrid nanostructures: future generation conducting materials, J. Mater. Chem. A 4 (2016) 9347-9361. 
[69] H. Yang, M.R. Villani, H. Wang, The role of cellular reactive oxygen species in cancer chemotherapy, J. Exp. Clin. Cancer Res. 37 (2018) 266:1-12.

[70] L. Huang, B. Wu, G. Yu, Graphene: learning from carbon nanotubes, J. Mater. Chem. 21 (2011) 919-929.
[71] B. Bilalis, D. Katsigiannopoulos, A. Avgeropoulos, Non-covalent functionalization ofcarbon nanotubes with polymers, RSC Adv. 4 (2014) 29112934.

[72] A.P.F. Turner, Biosensors: sense and sensibility, Chem. Soc. Rev. 42 (2013) 3184-3196. 